\title{
APROXIMACIÓN TEÓRICA DEL CONSTRUCTO E-UNIVERSIDAD BASADO EN UN ANÁLISIS SEMÁNTICO PRAGMÁTICO
}

\section{THEORETICAL APPROXIMATION OF THE E-UNIVERSITY CONSTRUCTION BASED ON A PRAGMATIC SEMANTIC ANALYSIS}

\begin{abstract}
Jesús Orlando Gómez Rivero, Ph.D. Doctor en Ciencias Humanas (Venezuela). Docente Titular Auxiliar a Tiempo Completo Universidad Iberoamericana del Ecuador y Director de Investigación (E) de la Universidad Iberoamericana del Ecuador, Quito, Ecuador. jgomez@unibe.edu.ec

Eddybelleth Yemala Castillo Brito, Ph.D. Doctora en Educación (Venezuela). Docente Titular Auxiliar a Tiempo Completo y Directora Académica de la Universidad Iberoamericana del Ecuador, Quito, Ecuador. ycastillo@unibe.educ.ec
\end{abstract}

\section{Ana Cecilia Quintana Arroyo, Mgs.}

Magíster en Telemática (Ecuador).

Docente Titular Auxiliar a Tiempo Completo y Jefe del Departamento de Tecnología (E) de la Universidad Iberoamericana del Ecuador, Quito, Ecuador. aquintana@unibe.edu.ec

\section{ARTÍCULO DE REFLEXIÓN}

Recibido: 8 de octubre de 2019.

Aceptado: 18 de diciembre de 2019.

\section{RESUMEN}

Desde la aparición de la sociedad del conocimiento, la e-universidad, como concepto inherente al uso de las TIC en las instituciones de educación superior ha sido poco tratado, 
debido a la falta de una visión sistémica y holística de la dinámica de la gestión universitaria, conjugada además con una orientación excesiva concentrada en la virtualización de la educación como su máxima referencia en los discursos sobre el tema. El estudio realizado fue de tipo documental de carácter teórico - conceptual que combinó el análisis crítico, el cotejo y la integración de perspectivas expuestas por diversos autores; utilizando para ello un análisis semántico pragmático. Desde el punto de vista metodológico, se estudió la construcción del concepto "e-gobierno" en todos los niveles; comenzando con la conceptualización de los aspectos tratados en el discurso, a partir de la matriz de conceptualización, donde se observó la perspectiva desde la cual los autores construyeron la imagen de la realidad presentada, con los resultados del análisis del término "e-gobierno" se construyó las perspectivas teóricas del término "e-universidad".

Palabras clave: Conectividad social, plataforma tecnológica, procesos, gestión.

\section{ABSTRACT}

Since the emergence of the knowledge society, e-university, as a concept inherent in the use of TIC in higher education institutions has been little treated, due to the lack of a holistic and systemic vision of the dynamics of university management, also combined with an excessive orientation focused on the virtualization of education as its maximum reference in speeches on the subject. The study was of a theoretical-conceptual documentary type that combined critical analysis, collation and integration of perspectives presented by various authors; using for this a pragmatic semantic analysis. From the methodological point of view, the construction of the "e-government" concept at all levels was studied; beginning with the conceptualization of the aspects treated in the discourse, from the conceptualization matrix, where the perspective from which the authors constructed the image of the presented reality was observed, with the results of the analysis of the term "e-government" The theoretical perspectives of the term "e-university" were built.

Keywords: Social connectivity, technology platform, processes, management. 


\section{INTRODUCCIÓN}

El desarrollo que han tenido en los últimos años las Tecnologías de Información y Comunicaciones (TIC) ha generado un fuerte impacto en los distintos ámbitos del quehacer de la sociedad, pues ha facilitado algunas actividades de la vida cotidiana y ha logrado mayor eficiencia y eficacia en el desarrollo organizacional. De allí que, el uso de las nuevas tecnologías, aunado con las herramientas de software, plataforma de hardware y redes de comunicación, se han generado una nueva forma de gestionar y ofrecer productos y/o servicios.

Este nuevo paradigma de la sociedad, conlleva a una inminente reconfiguración de las organizaciones para responder de forma efectiva a los retos del mundo globalizado, en donde la diversidad de actores y variables medioambientales, socioculturales, políticoadministrativas y tecno-científicas, entre otras, se abrazan en aras de una convivencia armónica. De allí la necesidad de las organizaciones de cualquier sector de reinventarse para responder con mayor efectividad es eminente, muy especialmente, aquellas en donde la interacción con el cliente y/o usuarios cobra su especial atención, revisión y dedicación de manera inmediata.

En este contexto nace el Gobierno Electrónico (e-government), que según Grosvald (2005), el concepto de "Gobierno Electrónico" incluye todas aquellas actividades basadas en las nuevas tecnologías informáticas, en particular Internet, que el Estado desarrolla para aumentar la eficiencia de la gestión pública, mejorar los servicios ofrecidos a los ciudadanos y proveer a las acciones del gobierno de un marco mucho más transparente que el actual, tanto en las aplicaciones internas como las externas de las tecnologías de la información y las comunicaciones (TIC) en el sector público.

Por lo que (e-government), tiene como objetivo hacer uso de las TIC para brindar mejores servicios al público usuario, así como mejorar la gestión interna de las instituciones. Generalmente, el término (e-government) se relaciona comúnmente con las estructuras relacionadas al Estado, sin embargo, existen organizaciones y/o instituciones que poseen su propio gobierno con amplia autonomía, en el cual el Estado, a través de leyes, establece 
su conformación básica de gobierno, lineamientos para su funcionamiento y mecanismos de control y seguimiento; tal es el caso de los Institutos de Educación Superior (IES). Por lo que se puede hablar de e-gobierno aplicado a las IES al cual se le puede acuñar el termino e-universidad.

En el marco de estos argumentos, esta investigación apunta hacia una aproximación teórica del constructo e-universidad, el cual está signado por la necesidad de asumir no solo nuevos paradigmas, sino nuevas racionalidades y prácticas para impulsar procesos de competitividad y de desarrollo social de los Institutos de Educación Superior.

\section{REVISIÓN TEÓRICA}

Sobre el análisis semántico-pragmático (Molero de Cabezas, 2003), sostiene que es un instrumento basado en una teoría y una metodología que permite acercarse al estudio de diferentes tipos de discursos, tomando en cuenta las formas lingüísticas. Asimismo, por ser semántico intenta explicar cómo se transforma el significado de las lenguas en significaciones contextualizadas y por ser pragmático, se interesa en la visión del discurso desde afuera, en el contexto; es decir, en un evento de comunicación.

Esta técnica se empleó para el desarrollo teórico conceptual acerca de los tópicos relacionados con el tema de estudio, ya que el análisis realizado permitió abordar sistemáticamente y descubrir más allá de lo superficial lo que está por debajo de todo este planteamiento y de esta manera facilitar la compresión de las posturas teóricas analizadas. En este contexto, el estudio llevado a cabo permitió conocer características generales y particulares del discurso científico, ya que el enfoque semántico-pragmático pone al descubierto algunos elementos, posiciones, instrumentos que con la simple lectura no se observa, pero con este análisis se puede sistematizar más adecuadamente.

El marco teórico que sustenta esta forma de análisis se construye desde el campo multidisciplinario del análisis del discurso. El componente semántico se apoya teóricamente en los principios de la semántica lingüística (Pottier, 1993), se enmarca en la corriente de análisis del discurso (Van Dijk, 1980, 1994, 1999), en los referentes teóricos de 
Charaudean, (2006) y en los resultados de las investigaciones en los estudios del discurso de Molero, (1985) y (2006) y Cabeza (1986) y (2005).

Las operaciones metodológicas utilizadas provienen de los referentes del enfoque semántico-pragmático en el análisis del discurso, propuesto por Molero de Cabezas (2003), León y Romero (2006), relacionados con el nivel lógico-conceptual, el nivel lingüístico y el nivel discursivo.

\section{MATERIALES Y MÉTODOS}

El presente estudio se desarrolló dentro de un tipo de investigación documental, que combinó el análisis crítico, el cotejo y la integración de perspectivas expuestas por diversos autores que han elaborado explicaciones acerca del constructo de estudio. En este sentido, la investigación es de carácter teórico - conceptual, ya que pretende conceptualizar, caracterizar y comprender los elementos que definen la e-universidad.

En relación con el enfoque metodológico, la investigación combinó diversos enfoques: Descriptivo porque se realizó el reconocimiento y caracterización de los elementos conceptuales que fundamentan la e-universidad; Explicativo porque se analizaron las características planteadas anteriormente; Evaluativo porque se reconstruyeron y valoraron los elementos del constructo partir del Análisis Semántico Pragmático.

\section{RESULTADOS}

Para la construcción de los elementos teóricos-conceptuales que fundamentan la euniversidad, es necesario establecer la relación teórica práctica con el constructo egobierno debido que la mayoría de los trabajos de investigación y la práctica misma de los gobiernos han contribuido a avanzar el estado del arte del mismo, por lo que poco se ha avanzado en la aplicación de los conceptos, métodos, herramientas y prácticas de gobierno electrónico al ámbito universitario.

Cabe hacer notar que, la mayor parte de los documentos producidos por organismos 
internacionales y diferentes autores está orientado a el e-gobierno, por lo que la construcción del constructo "e-universidad" partió con el análisis del alcance conceptual del término "e-gobierno", para lo cual se seleccionaron las perspectivas teóricas acerca del egobierno expuestas por los autores considerados con mayor presencia y representatividad en internet, se empleó el método de análisis semántico-pragmático, el cual permitió develar los elementos conceptuales comunes entre uno y otro autor y de allí derivar los rasgos que definen a la e-gobierno y construir a partir de esto dicho constructo.

Desde el punto de vista metodológico, se estudió la construcción del concepto "e-gobierno" en todos los niveles; comenzando con la conceptualización de los aspectos tratados en el discurso, a partir de la matriz de conceptualización, donde se observó la perspectiva desde la cual los autores construyeron la imagen de la realidad presentada. Las perspectivas analizadas se presentan en el Tabla 1.

Tabla 1. Perspectivas teóricas acerca de la "e-gobierno".

\begin{tabular}{|l|l|l|}
\hline \multicolumn{1}{|c|}{ AUTOR } & AÑO & \multicolumn{1}{|c|}{ PERSPECTIVA TEÓRICA } \\
\hline WASSENAAR & $\mathbf{2 0 0 0}$ & $\begin{array}{l}\text { Desde una perspectiva de empresa, es la } \\
\text { aplicación de las TIC para mejorar, transformar o } \\
\text { redefinir cualquier forma de intercambio de } \\
\text { recursos e información entre compañías, } \\
\text { agencias gubernamentales, ciudadanos, } \\
\text { proveedores y otros participantes, desarrollando } \\
\text { y manteniendo sistemas inter organizacionales, } \\
\text { organizaciones virtuales y acuerdos } \\
\text { interinstitucionales. }\end{array}$ \\
\hline WOHLLEBEN & \multirow{2}{*}{$\begin{array}{l}\text { El gobierno electrónico no es simplemente } \\
\text { asumir la tecnología. Es un estado mental que } \\
\text { implica comunicar al ciudadano con el gobierno } \\
\text { para obtener ciertos objetivos. La transformación } \\
\text { es lo más importante, aún por encima de los } \\
\text { servicios. El impacto real es la transformación en } \\
\text { un gobierno diferente, difícil de alcanzar, que } \\
\text { provea servicios modernos y diferentes de los } \\
\text { que actualmente se están proveyendo. }\end{array}$} \\
\hline
\end{tabular}




\begin{tabular}{|c|c|c|}
\hline AUTOR & AÑO & PERSPECTIVA TEÓRICA \\
\hline $\begin{array}{c}\text { TAMBOURIS, E., } \\
\text { GORILAS, S., \& BOUKIS, } \\
\text { G. }\end{array}$ & 2001 & $\begin{array}{l}\text { Es la aplicación de las TIC para transformar la } \\
\text { eficiencia, eficacia, transparencia, } \\
\text { responsabilidad de los intercambios de } \\
\text { transacciones e información entre agencias } \\
\text { gubernamentales y entre el gobierno y el } \\
\text { ciudadano o empresas, además del } \\
\text { empoderamiento del ciudadano a través del } \\
\text { acceso y uso de la información. }\end{array}$ \\
\hline $\begin{array}{l}\text { ORGANIZACIÓN DE } \\
\text { ESTADOS } \\
\text { AMERICANOS (OEA) }\end{array}$ & 2003 & $\begin{array}{l}\text { Es un compromiso permanente del Gobierno } \\
\text { para mejorar la relación de los ciudadanos y la } \\
\text { Administración pública, mediante el intercambio } \\
\text { eficaz y eficiente de servicios, información y } \\
\text { conocimiento. }\end{array}$ \\
\hline BONNEFOY & 2004 & $\begin{array}{l}\text { Es la capacidad que ofrecen las TIC para } \\
\text { desarrollar y ofrecer información y servicios } \\
\text { públicos a través de medios telemáticos, } \\
\text { habitualmente Internet. }\end{array}$ \\
\hline ESTEVES & 2005 & $\begin{array}{l}\text { Es un proceso que implica un cambio } \\
\text { transformacional a nivel organizacional, en este } \\
\text { sentido el gobierno electrónico es más que una } \\
\text { tecnología, más que internet, más que una } \\
\text { entrega de servicios, es acerca de poner los } \\
\text { ciudadanos y clientes en el corazón de todo lo } \\
\text { que hacemos y construir el acceso a servicios, } \\
\text { su entrega y responsabilidad democrática a su } \\
\text { alrededor. }\end{array}$ \\
\hline $\begin{array}{l}\text { INSTITUTO DE LAS } \\
\text { AMÉRICAS }\end{array}$ & 2006 & $\begin{array}{l}\text { Es un proceso que colabora en la aceleración } \\
\text { del tránsito desde una administración } \\
\text { autocentrada a una centrada en el ciudadano, } \\
\text { mejorando la calidad de los servicios y facilitando } \\
\text { el cumplimiento de las obligaciones de los } \\
\text { ciudadanos y organizaciones con el Estado. }\end{array}$ \\
\hline MONTILLA & 2007 & $\begin{array}{l}\text { Es un modelo interactivo de gestión pública, } \\
\text { basado en el empoderamiento de los ciudadanos } \\
\text { y las organizaciones a través del uso con sentido } \\
\text { de las Tecnologías de la Información y la } \\
\text { Comunicación. }\end{array}$ \\
\hline
\end{tabular}

Fuente: Elaboración propia.

Para la comprensión de las definiciones se elaboró una matriz de conceptualización propuesta por Molero de Cabezas (2003), la cual facilitó el análisis de las perspectivas desde las cuales los autores construyen el concepto de "e-gobierno" (Ver Tabla 2). 
Considerando los elementos que se consideraron son los siguientes:

a. Visión de la situación: referida a como el autor centraliza la idea fundamental acerca del tema estudiado.

b. Causantes: está relacionada con los elementos que generan o activan la situación de cambio.

c. Tipo de cambio: es la situación generada por la actuación de los causantes.

d. Instrumento del cambio: son todos aquellos elementos y herramientas que facilitan el cambio.

e. Agentes del cambio: son los individuos u organizaciones que generan el proceso planteado. 
Tabla 2. Concepción de "e-gobierno" en el nivel lógico-conceptual.

\begin{tabular}{|c|c|c|c|c|c|}
\hline & $\begin{array}{l}\text { Visión de la situación de } \\
\text { "e-gobierno" }\end{array}$ & Causantes & Tipo de Cambio & $\begin{array}{l}\text { Instrumento } \\
\text { del cambio }\end{array}$ & Agentes del Cambio \\
\hline WASSENAAR & $\begin{array}{l}\text { Intercambio de recursos e } \\
\text { información entre todas las partes } \\
\text { interesadas de la organización. }\end{array}$ & $\begin{array}{l}\text { Necesidad de los ciudadanos de } \\
\text { acceso a información. }\end{array}$ & $\begin{array}{l}\text { Mejora en la prestación de } \\
\text { servicios a sus ciudadanos. }\end{array}$ & TIC & $\begin{array}{l}\text { Implica innovación en las relaciones } \\
\text { internas y externas del Gobierno con otras } \\
\text { agencias gubernamentales, con sus } \\
\text { propios empleados, con las empresas y } \\
\text { con el ciudadano, particularmente } \\
\text { aplicaciones relacionadas con Internet. }\end{array}$ \\
\hline WOHLLEBEN & $\begin{array}{l}\text { Comunicación del ciudadano con el } \\
\text { gobierno para obtener ciertos } \\
\text { objetivos. }\end{array}$ & Necesidad de los ciudadanos. & $\begin{array}{l}\text { Transformación en un } \\
\text { gobierno diferente que } \\
\text { provea servicios modernos. }\end{array}$ & TIC & $\begin{array}{l}\text { Transformación en la prestación del } \\
\text { servicio administrativos del gobierno. }\end{array}$ \\
\hline $\begin{array}{l}\text { TAMBOURIS, E., } \\
\text { GORILAS, S., \& } \\
\text { BOUKIS, G. }\end{array}$ & $\begin{array}{l}\text { Transformación de los } \\
\text { intercambios de transacciones e } \\
\text { información entre agencias } \\
\text { gubernamentales y entre el } \\
\text { gobierno y el ciudadano o } \\
\text { empresas. }\end{array}$ & $\begin{array}{l}\text { Exigencia del entorno en la } \\
\text { mejora de los servicios del } \\
\text { Gobierno. }\end{array}$ & $\begin{array}{l}\text { Empoderamiento del } \\
\text { ciudadano a través del } \\
\text { acceso y uso de la } \\
\text { información. }\end{array}$ & TIC & $\begin{array}{l}\text { Ciudadanos con fácil acceso a la } \\
\text { información y servicios administrativos del } \\
\text { estado. }\end{array}$ \\
\hline BONNEFOY & $\begin{array}{l}\text { Suministro de información y } \\
\text { servicios públicos a través de } \\
\text { medios telemáticos. }\end{array}$ & Exigencias del entorno. & $\begin{array}{l}\text { Plataformas de información } \\
\text { de fácil acceso. }\end{array}$ & TIC & $\begin{array}{l}\text { Transformación en el acceso de } \\
\text { información y servicios. }\end{array}$ \\
\hline $\begin{array}{l}\text { ORGANIZACIÓN } \\
\text { DE ESTADOS } \\
\text { AMERICANOS } \\
\text { (OEA) }\end{array}$ & $\begin{array}{l}\text { Mejoramiento de la relación de los } \\
\text { ciudadanos y la Administración } \\
\text { pública, mediante el intercambio } \\
\text { eficaz y eficiente de servicios, } \\
\text { información y conocimiento. }\end{array}$ & Necesidad de los ciudadanos. & $\begin{array}{l}\text { Ciudadanos con acceso a } \\
\text { servicios, información y } \\
\text { conocimiento de forma } \\
\text { efectiva. }\end{array}$ & TIC & $\begin{array}{l}\text { Procesos eficaces y eficientes en la } \\
\text { generación y acceso de información. }\end{array}$ \\
\hline ESTEVES & $\begin{array}{l}\text { Poner los ciudadanos y clientes en } \\
\text { el corazón de todo lo que hacemos } \\
\text { y construir el acceso a servicios, su }\end{array}$ & Exigencias del entorno. & $\begin{array}{l}\text { Ciudadanos con acceso a } \\
\text { servicios, información y }\end{array}$ & TIC & $\begin{array}{l}\text { Cambio transformacional a nivel } \\
\text { organizacional. }\end{array}$ \\
\hline
\end{tabular}

ISSN: 1390-9320, Edición Especial, febrero 2020 
Revista Científica ECOCIENCIA

\begin{tabular}{|c|c|c|c|c|c|}
\hline & $\begin{array}{l}\text { Visión de la situación de } \\
\text { "e-gobierno" }\end{array}$ & Causantes & Tipo de Cambio & $\begin{array}{l}\text { Instrumento } \\
\text { del cambio }\end{array}$ & Agentes del Cambio \\
\hline & $\begin{array}{l}\text { entrega y responsabilidad } \\
\text { democrática a su alrededor. }\end{array}$ & & $\begin{array}{l}\text { conocimiento de forma } \\
\text { efectiva. }\end{array}$ & & \\
\hline $\begin{array}{l}\text { INSTITUTO DE } \\
\text { LAS AMÉRICAS }\end{array}$ & $\begin{array}{l}\text { Mejora de la calidad de los } \\
\text { servicios y cumplimiento de las } \\
\text { obligaciones de los ciudadanos y } \\
\text { organizaciones con el Estado. }\end{array}$ & $\begin{array}{l}\text { Exigencia del entorno en la } \\
\text { mejora de los servicios del } \\
\text { Gobierno. }\end{array}$ & $\begin{array}{l}\text { Ciudadanos con acceso a } \\
\text { servicios, información y } \\
\text { conocimiento y cumplimiento } \\
\text { oportuno de las obligaciones. }\end{array}$ & TIC & $\begin{array}{l}\text { Desde una administración autocentrada a } \\
\text { una centrada en el ciudadano. }\end{array}$ \\
\hline MONTILLA & $\begin{array}{l}\text { Modelo interactivo de gestión } \\
\text { pública. }\end{array}$ & $\begin{array}{l}\text { Exigencia del entorno en la } \\
\text { mejora de los servicios del } \\
\text { Gobierno. }\end{array}$ & $\begin{array}{l}\text { Empoderamiento de los } \\
\text { ciudadanos y las } \\
\text { organizaciones. }\end{array}$ & TIC & $\begin{array}{l}\text { Transformación en el acceso de } \\
\text { información y servicios. }\end{array}$ \\
\hline
\end{tabular}

Fuente: Elaboración propia.

ISSN: 1390-9320, Edición Especial, febrero 2020 
De acuerdo a las posturas de cada autor sobre "e-gobierno" se puede inferir que los mismos visualizan los siguientes elementos:

1. Se establece como instrumento que potencian el desarrollo del e-gobierno el uso efectivo de las TIC e implica innovación en las relaciones internas y externas del Gobierno con todas las partes interesadas.

2. Está relacionado principalmente con lo relativo al acceso y a la provisión de información gubernamental y a la prestación de servicios a sus ciudadanos.

3. Busca optimizar el uso de los recursos para el logro de los objetivos gubernamentales.

4. Su implementación implica una trasformación en todos los procesos y la visión de prestación de servicios.

5. Es un medio, no un fin en sí mismo.

6. Alude al cambio en la forma en que se gestiona la Administración Pública, nuevos sistemas, eficiencia, eficacia, mejoras en la gestión entre otros.

En el Tabla 3 se presenta el Tabla resumen del análisis.

Tabla 3. Resumen de la concepción de "e- gobierno".

\begin{tabular}{|l|l|}
\hline $\begin{array}{l}\text { Visión de la situación de "e- } \\
\text { gobierno" }\end{array}$ & $\begin{array}{l}\text { Intercambio de recursos e información entre } \\
\text { todas las partes interesadas del gobierno. }\end{array}$ \\
\hline Los causantes & $\begin{array}{l}\text { Necesidad y dinámica del entorno. } \\
\text { Revolución tecnológica. }\end{array}$ \\
\hline Tipo de cambio & $\begin{array}{l}\text { Empoderamiento del ciudadano a través del } \\
\text { acceso y uso de la información. }\end{array}$ \\
\hline Instrumento del cambio & TIC \\
\hline Los Agentes del Cambio & $\begin{array}{l}\text { Transformación en la prestación del servicio } \\
\text { administrativos del gobierno. }\end{array}$ \\
\hline
\end{tabular}

Fuente: Elaboración propia.

Finalmente, se puede afirmar que el e-gobierno vincula estrechamente al Estado, la gestión pública, la sociedad, el ciudadano y las TIC hacia el desarrollo de políticas y el fortalecimiento de la sociedad del conocimiento como sistema económico social. Al respecto Katz y Hilbert (2003) señalan que, en este ámbito de integralidad, la Comisión 
Económica para América Latina y el Caribe (CEPAL) definió el marco conceptual mediante el cual es posible establecer las relaciones más importantes de este sistema, el cual engloba dos elementos a saber: la infraestructura y servicios genéricos (software, navegadores, almacenamiento, Internet) los cuales actúan de forma transversal a los diferentes estratos sociales que conforma el sistema (e-gobierno, e-salud, e-negocios, e-universidad, entre otros) y son impactados por: un marco regulatorio, financiación y capital humano los cuales fortalecen el desarrollo de la del sistema, tal como se muestra en la Figura 1.

Figura 1. Componentes del e-gobierno.

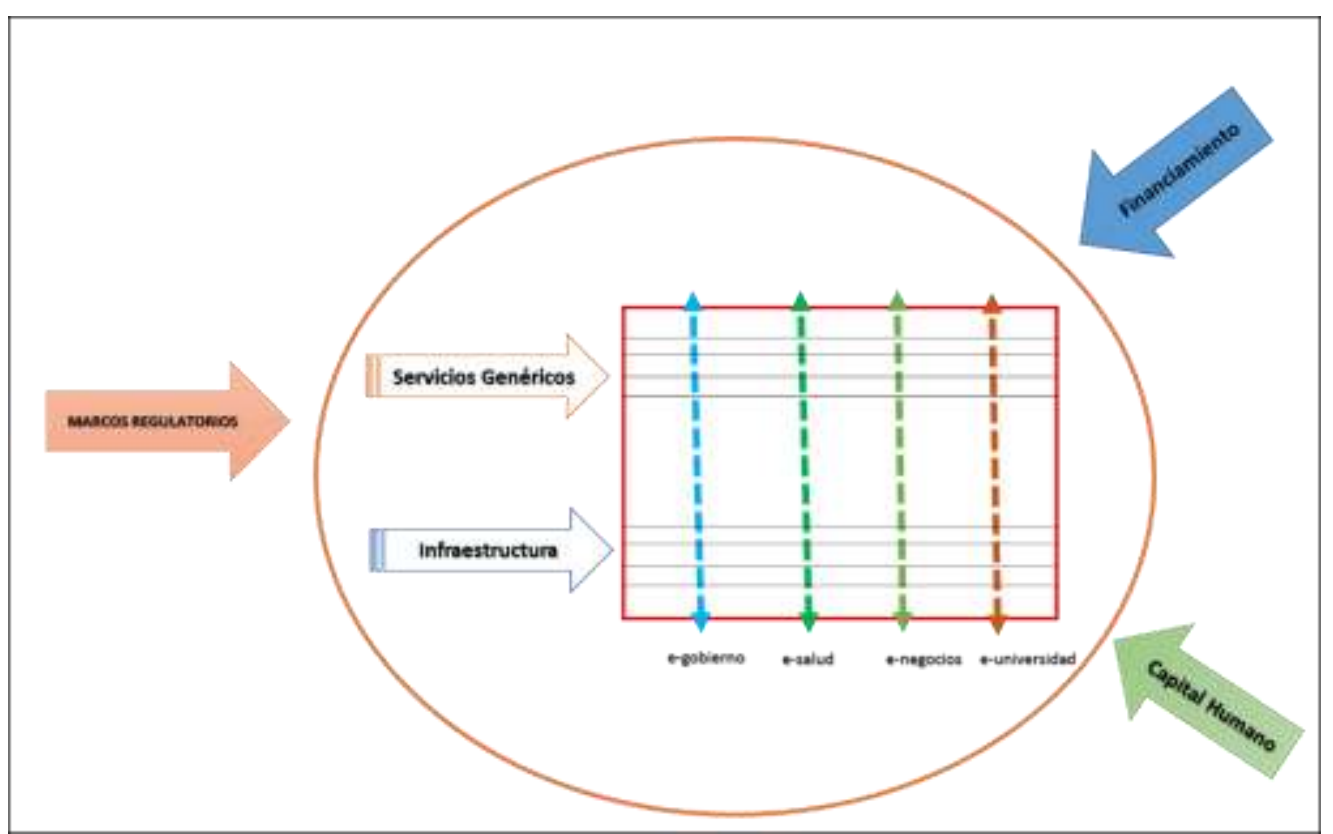

Fuente: Elaboración propia de Gómez, Castillo y Quintana (2018) a partir de CEPAL.

En este fortalecimiento, el e-gobierno involucra a todos los entes del Estado en la configuración de políticas integrales, tanto a nivel nacional, estatal y municipal; por lo cual, según la UNESCO (1998) se requiere que el e-gobierno defina políticas por sector orientadas a la integración de estos entes y la necesaria digitalización de cada uno, incluida el concepto de universidad como instancia pública educativa.

Dentro de este contexto las universidades requieren desarrollar mecanismos estratégicos que le permitan mejorar los servicios que presta aprovechando el auge del conocimiento y las nuevas tecnologías en el marco de la sociedad del conocimiento y 
es lo va constituir el concepto de "e- universidad" que surge con la intención de utilizar las nuevas tecnologías de información y comunicación para compartir y gestionar el conocimiento y ofrecer una mejor comunicación a los docentes, alumnos, personal administrativo, visitantes, investigadores, entes intergubernamentales y comunidad en general.

Entonces en función de relación teórica práctica a partir de con los resultados del análisis del término "e-gobierno", las perspectivas teóricas del término "e-universidad" se resumen en una visión de servicio público dentro del sistema de conectividad social y su relación estrecha con los e-usuarios y el e-servicio, a partir de una plataforma tecnológica explícitamente diseñada, bajo principios de calidad, responsabilidad y pertinencia en sus procesos de gestión, docencia, investigación, vinculación; dentro de un marco adecuado de normas, financiación y capital humano.

Finalmente, fundamentado en el marco conceptual que sustenta el e-gobierno la euniversidad entonces podría conceptualizada a partir de la interrelación de los siguientes componentes: el e-gobierno universitario, la e-docencia, la e-investigación, la evinculación y el e-servicio; los cuales son impactados de forma transversal por la infraestructura y el servicio genérico y dirigido por un capital humano; todo esto dentro de un marco regulatorio que guía su funcionamiento. Esta concepción se presenta en la Figura 2. 
Figura 2. Componentes de la e-universidad.

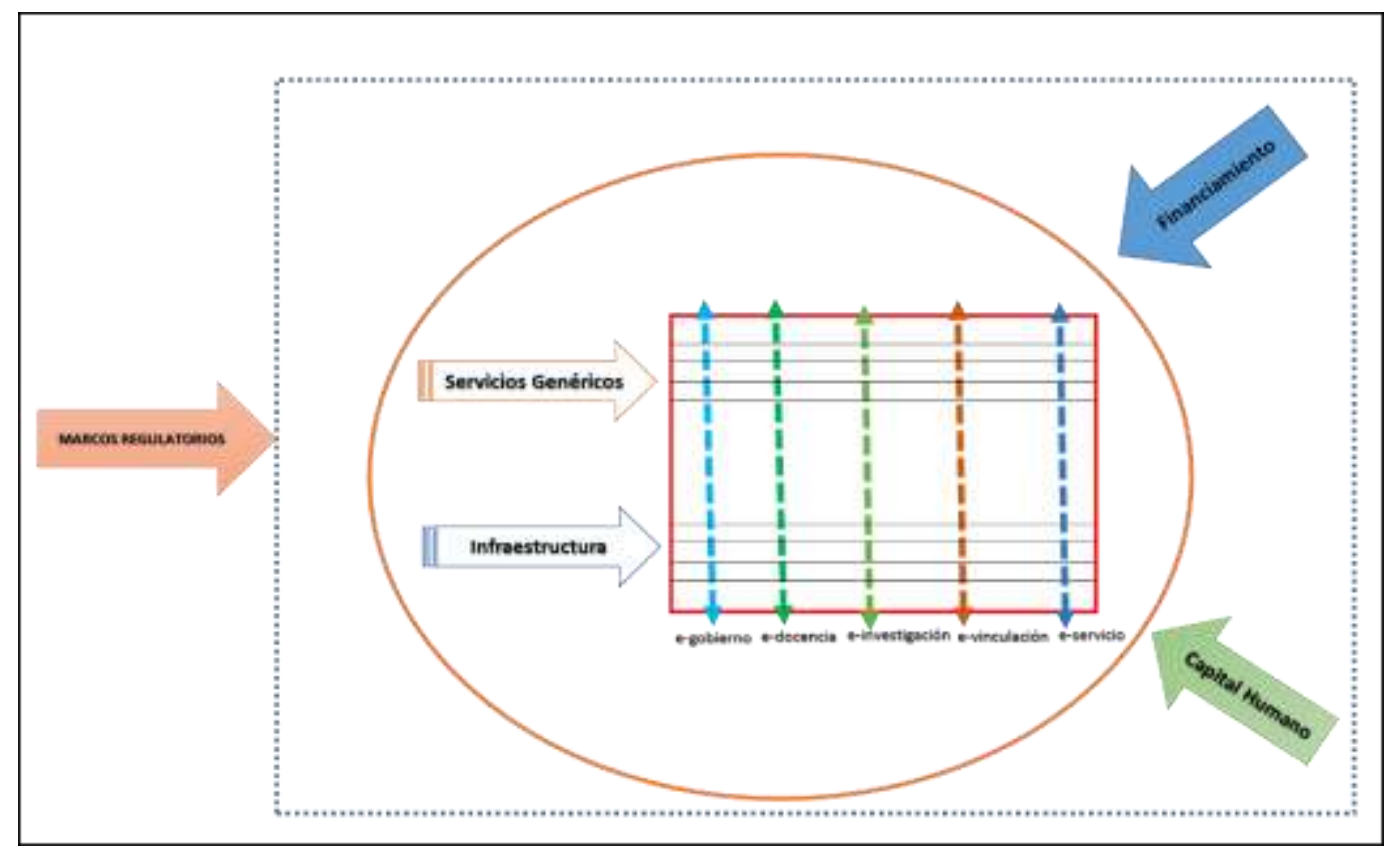

Fuente: Elaboración propia de Gómez, Castillo y Quintana (2018) a partir de CEPAL.

\section{CONCLUSIONES Y RECOMENDACIONES}

La e-universidad se origina desde una visión de servicio público dentro del sistema de conectividad social, fundamentada sobre una interrelación vital de la interrelación de los siguientes componentes: el e-gobierno universitario, la e-docencia, la e-investigación, la e-vinculación y el e-servicio; los cuales son impactados de forma transversal por la infraestructura y el servicio genérico y dirigido por un capital humano; todo esto dentro de un marco regulatorio que guía su funcionamiento.

Es importante recalcar que, la e-universidad no cambia el concepto clásico de una universidad como productora de conocimiento, valores y centro de discusión científica por excelencia, sino que permite una mejor conexión con la sociedad y el desarrollo de ésta en su conjunto.

\section{REFERENCIAS BIBLIOGRÁFICAS}

Bonnefoy, J. (2004). Gestión Pública y Gobierno Electrónico. Instituto latinoamericano del Caribe de Planificación Económica y Social ILPES/CEPAL. 
Recuperado

https://repositorio.cepal.org/bitstream/handle/11362/7330/1/S1100145_es.pdf

Esteves, J. (2005). Análisis del Desarrollo del Gobierno Electrónico Municipal en España, Madrid, octubre de 2005. Papel de Trabajo, Cátedra Software AG Alianza Sumaq en eGovernment.

Grosvald, G. (2005). Revista electrónica "datos en la red.com”

Instituto de las Américas (2006). Consideraciones para la Creación de una Nueva Agenda Mexicana de Gobierno Electrónico para el Próximo Sexenio. Recuperado de: http://www.iamericas.org/pdfs/RoundtableReports/InformeFinalde-e-Gobierno2006.pdf

Katz, J. y Hilbert, M. (2003). Los Caminos Hacia una Sociedad de la Información en América Latina y el Caribe. ONU/CEPAL.

Molero, L. (2003). El enfoque semántico-pragmático en el análisis del discurso. Visión teórica actual. Lingua americana, N. 7, Vol.12 p.p.5-28.

Montilla, A. y A. Páez (2010). Experiencias de participación ciudadana a través de internet en centros de gestión parroquial de Venezuela. Quórum Académico. Vol. 7, Núm. 1, 11-44

Organización de Estados Americanos (OEA) (2003). Agenda para la Conectividad de las Américas. Plan de Acción de Quito [documento en línea]. OEA. Recuperado de: http://www.citel.oas.org/sp/Connectividad/ Final\%20Spanish\%20ACAPAO-march-5-2003-v3_e.pdf

Tambouris, E., Gorilas, S., \& Boukis, G. (2001). Investigation of Electronic Government. Atenas: Archetypon S.A.

UNESCO (1998). Declaración Mundial sobre la Educación Superior en el siglo XXI: Visión y Acción. Unesco 
Wassenaar, A. (2000). E-Govermental Value Chain Models. (pp. 289-293). London, Greenwich: DEXA 2000, IEEE Press citado por Cardona MBA. (2002) "El gobierno electrónico: Una revisión desde la perspectiva de la prestación de servicios".

Wohlleben, P. (2001). (Partner in the global government group of Grant Thornton LLP of Chicago). What's E-Government? T. TEMIN (GCN editorial director), "What's E-Government? How Do We Do It?" (p. 8). Government Computer News citado por Cardona MBA. (2002) "El gobierno electrónico Una revisión desde la perspectiva de la prestación de servicios". 